\title{
ANÁLISIS DEL DESEMPEÑO DE UN SISTEMA DE COMUNICACIONES CON MODULACIÓN 16/64 QAM BASADO EN HARDWARE RECONFIGURABLE
}

\author{
PERFORMANCE ANALYSIS OF A COMMUNICATION SYSTEM OF 16/64 QAM MODULA- \\ TION BASED ON RECONFIGURABLE HARDWARE
}

DESEMPEÑO DE UN SISTEMA DE COMUNICACIONES SOBRE HARDWARE RECONFIGURABLE

Juan Camilo Zemanate Zuñiga' ${ }^{1}$, Julián Andrés Muñoz Hidalgo ${ }^{2}$, Victor Manuel Quintero Flórez ${ }^{3}$

$\begin{array}{r}\text { 1. Universidad del Cauca Popayán, Colombia } \\ \text { 2. Universidad del Cauca Popayán, Colombia } \\ \text { 3. Universidad del Cauca Popayán, Colombia }\end{array}$
$\begin{array}{r}\text { Recibido: } 07 \text { de febrero de 2014 } \\ \text { Aceptado: } 08 \text { de marzo de 2014 } \\ \text { Correspondencia relativa: Juan Camilo Zemanate Zuñiga. Cra 3 \# 7-33 Popayán (C.) juanzemanate@unicauca.edu.co }\end{array}$

\section{RESUMEN}

En el presente artículo se analiza el desempeño de un sistema de comunicaciones banda base con Modulación de Amplitud en Cuadratura (QAM, Quadrature Amplitude Modulation), implementado sobre un Arreglo de Compuertas Programables de Campo (FPGA, Field Programmable Gate Arrays), a partir de la Tasa de Error de Bit (BER, Bit Error Rate) y del comportamiento del hardware reconfigurable.

El sistema de comunicaciones banda base consideró un canal de Ruido Blanco Gaussiano Aditivo (AWGN, Additive White Gaussian Noise) y las modulaciones 16 QAM y 64 QAM. Se diseñó a través de la herramienta System Generator ${ }^{\circledR}$ de Xilinx ${ }^{\circledR}$, se validó (a nivel de simulación) por medio de la comparación con un modelo en Simulink ${ }^{\circledR}$ y se implementó sobre un FPGA Spartan 6 de Xilinx ${ }^{\circledR}$ mediante la descarga de un archivo de programación. Como resultados se obtuvieron las curvas de desempeño del sistema de comunicaciones banda base con modulación 16/64 QAM, las cuales fueron comparadas con las curvas de desempeño teóricas ofrecidas por la herramienta Bertool ${ }^{\circledR}$ de Matlab ${ }^{\circledR}$ para su correspondiente análisis. De este modo, se concluyó que dada la similitud de las curvas de desempeño obtenidas a partir de System Generator ${ }^{\circledR}$, Simulink ${ }^{\circledR}$, implementación y Bertool ${ }^{\circledR}$, el sistema de comunicaciones banda base alcanzó resultados óptimos sobre hardware reconfigurable.

Palabras clave: Comunicaciones banda base, FPGA, Hardware reconfigurable, Modulación QAM, System Generator®. 


\begin{abstract}
In this article, the performance of a baseband communications system of Quadrature Amplitude Modulation (QAM) implemented on a Field Programmable Gate Arrays (FPGA) is analyzed from the Bit Error Rate (BER) and the behavior of reconfigurable hardware. The baseband communications system considered an Additive White Gaussian Noise (AWGN) and the 16 QAM and 64 QAM modulations. It was designed through the Xilinx ${ }^{\circledR}$ System Generator ${ }^{\circledR}$ tool, was validated (at a simulation level) by means of a comparison with a model in Simulink ${ }^{\circledR}$ and was implemented on FPGA Spartan 6 of Xilinx ${ }^{\circledR}$ by downloading a programming file. As results, the basic performance curves of the baseband communications system of 16/64 QAM modulation were obtained and they were compared to the theoretical performance curves provided by the Matlab ${ }^{\circ}$ Bertool ${ }^{\circledR}$ tool for its corresponding analysis. Thus, it was concluded that given the similarity of the performance curves obtained from System Generator ${ }^{\circledR}$, Simulink $\AA$, implementation and Bertool $\AA$, the communication system achieved outstanding results upon reconfigurable hardware..
\end{abstract}

Keywords-Base Band Communications, FPGA, QAM Modulation, Reconfigurable Hardware, System Generator®.

\section{INTRODUCCIÓN}

Ante los elevados costos de implementación de un sistema de comunicaciones sobre hardware convencional, existe la necesidad de contar con un sistema hardware flexible y reconfigurable que permita una disminución significativa de los costos de implementación y que a su vez posibilite la evaluación del desempeño a nivel físico de los diversos esquemas de modulación en un sistema de comunicaciones real.

Aunque se ha desarrollado un sinnúmero de tecnologías de hardware reconfigurable, la tecnología FPGA representa hoy en día la tecnología de mayor acogida, pues aunque es una tecnología ya madura sus avances continúan aumentando la cantidad de compuertas lógicas y memoria disponible, brindando una mayor posibilidad de integración de funciones que van desde simples multiplicadores cableados, pasando por transmisores-receptores de alta velocidad hasta llegar a núcleos de Unidades Centrales de Procesamiento (CPU, Central Processing Unit) con periféricos asociados.

Así, la implementación de sistemas de comunicaciones completos sobre un único FPGA se hace posible y además de todo, sencillo con las mejores condiciones en consumo de potencia, desempeño, fiabilidad y costo [1].

Adicional a lo anterior, hoy en día los fabricantes de FPGAs proporcionan, además del hardware, herramientas software que facilitan el diseño y síntesis de modelos de sistemas de comunicaciones; este es el caso de System Generator ${ }^{\circledR}$, una herramienta de alto nivel para el diseño de sistemas sobre FPGAs de Xilinx ${ }^{\circledR}$ que funciona dentro del modelado y simulación del ambiente de Simulink ${ }^{\circledR}$. Su principal ventaja radica en la posibilidad de combinar bloques funcionales de Xilinx ${ }^{\circledR}$ con Matlab ${ }^{\circledR}$ y Simulink ${ }^{\circledR}$ para crear un banco de pruebas realistas y analizar así los datos generados por el modelo. El alto nivel de abstracción proporcionado por System Generator ${ }^{\circledR}$ simplifica en gran medida el desarrollo de algoritmos y su verificación incluso en sistemas de comunicaciones multifrecuencia sofisticados.

Así, las librerías de Xilinx ${ }^{\circledR}$ que incluyen bloques de comunicación, lógica de control, procesamiento de señales, matemáticas y memoria, permiten integrar código HDL, funciones de Matlab ${ }^{\circledR}$ y componentes de hardware diseñados para FPGAs de Xilinx ${ }^{\circledR}$, con el objetivo de crear modelos de sistemas de comunicaciones completos que se puedan simular en el entorno Simulink $\AA$.

En razón a lo anteriormente expuesto, considerando el gran potencial que representa el uso de FPGAs como hardware reconfigurable y destacando las ventajas de diseño y síntesis que proporciona el software de diseño de alto nivel para la implementación de sistemas de comunicaciones, y por tanto su aplicación en esquemas de modulación, en el presente artículo se analiza el desempeño de un sistema de comunicaciones banda base con modulación 16/64 QAM y un canal AWGN, implementado sobre FPGA, haciendo 
uso de la herramienta System Generator ${ }^{\circledR}$ ofrecida por Xilinx ${ }^{\circledR}$.

\section{SISTEMA DE COMUNICACIONES DIGITAL}

Los sistemas de comunicaciones pueden ser clasificados en dos grupos dependiendo del rango de frecuencias utilizadas para la transmisión de información: banda base y pasa banda.

Un sistema de comunicaciones digital banda base, envía la señal de información en su forma original en términos de frecuencia (sin traslación en el dominio de la frecuencia) y convierte la señal de información en formas de onda compatibles con las características del canal de comunicaciones; por su parte, un sistema de comunicaciones pasa banda, además de hacer la correspondiente conversión a formas de onda, realiza desplazamiento de la señal de información a una frecuencia mucho más alta que la frecuencia banda base (frecuencia portadora fc que resulta ser mucho más grande que la máxima componente de la señal banda base), brindando así mejores condiciones [2] a la señal de información al momento de propagarse por el canal de comunicaciones.

Al proceso completo de conversión a formas de onda y traslación en frecuencia se le conoce como modulación [3].

\section{A. Modulación Digital}

La modulación digital corresponde al proceso mediante el cual una secuencia de bits, que representa la señal de información, es convertida en formas de onda apropiadas para ser transmitidas sobre un canal de comunicaciones [3]. Este proceso consiste en la variación sistémica de uno o más parámetros (amplitud, frecuencia o fase) de una señal portadora analógica sinusoidal de acuerdo a la señal de información.

Las modulaciones digitales se clasifican en modulaciones binarias y modulaciones multinivel según el número de posibles estados (símbolos) que pueda tomar la señal portadora para representar la señal de información. En el caso de las modulaciones binarias el número símbolos es igual dos, lo que implica el uso de un solo bit por símbolo; en el caso de las modulaciones multinivel el número de símbolos es igual a $\mathrm{m}$, lo que implica el uso de $\log _{2} \mathrm{~m}$ bits por símbolo. Ya que la cantidad de bits por símbolo transmitido es mayor en la modulación multinivel, esta modulación supone un incremento en la velocidad de transmisión de datos frente a la modulación binaria.

\section{B. Modulación QAM}

La modulación QAM es una técnica de modulación digital multinivel cuya información está contenida tanto en la amplitud como en la fase de la señal que se transmite. Esencialmente se transmiten dos señales de información independientes por un único camino. Esto se consigue modulando una misma portadora, desfasada $90^{\circ}$ entre una señal de información y la otra [4]. La modulación QAM cuenta con dos etapas básicas, una etapa banda base y una etapa pasa banda.

\section{1) Etapa banda base}

En esta etapa una secuencia de bits es convertida en una secuencia de números complejos y la secuencia de números complejos es transformada en formas de onda adecuadas a las características del canal de comunicaciones [3]. La salida de la etapa banda base se expresa en (1).

$$
u(t)-\sum_{n=-\infty}^{\infty} a[n] p(t-n T)
$$

Donde,

$\mathrm{u}(\mathrm{t})$, salida de la etapa banda base.

$\mathrm{a}[\mathrm{n}]$, secuencia de símbolos complejos.

$\mathrm{p}(\mathrm{t}-\mathrm{nT})$, pulsos desplazados entre si T segundos, representan las formas de onda.

\section{2) Etapa pasa banda}

En esta etapa, la salida banda base $\mathrm{u}(\mathrm{t})$ es trasladada a una frecuencia fc. Para lograrlo, $u(t)$ es multiplicada por $e^{2 \pi j f_{c} t}$ originando una forma de ond a $u(t) e^{2 \pi j f_{c} t} \quad$ de carácter complejo, por lo cual se adiciona su conjugado complejo para convertirla en una forma de onda real, como resultado se obtiene la salida de la etapa pasa banda expresada en (2) [3].

$$
\begin{aligned}
& x(t)=u(t) e^{2 \pi j f_{c} t}+u(t)^{*} e^{-2 \pi j f_{c} t} \\
= & 2 \Re\{u(t)\} \cos \left(2 \pi f_{c} t\right)-2 \Im\{u(t)\} \operatorname{sen}\left(2 \pi f_{c} t\right)
\end{aligned}
$$


Donde,

$x(t)$, salida de la etapa pasa banda. $u(t)$, salida de la etapa banda base. $u(t)^{*}$, conjugado complejo de $u(t)$. $\mathfrak{R}\{\ldots\}$, operador parte real.

\section{$\mathfrak{I}\{\ldots\}$, operador parte imaginaria.}

Como lo presenta (2), las componentes real e imaginaria de la salida banda base son multiplicadas por una misma portadora desfasada $90^{\circ}$ entre una componente y la otra, obteniéndose dos señales ortogonales entre sí que pueden ser mezcladas sin temor a perderse la capacidad de distinguir entre una y otra.

\section{METODOLOGÍA}

Para la construcción del sistema de comunicaciones banda base sobre hardware reconfigurable (FPGA) se empleó el Modelo Lineal Secuencial (MLS) [5], en el cual se consideraron las etapas de modelado, simulación e implementación. En el modelado se exploró conceptualmente la obtención de un modelo para el sistema de comunicaciones banda base con modulación 16/64 QAM y un canal AWGN. En la simulación se consideró la selección de la herramienta software, la simulación del sistema de comunicaciones modelado y la validación del sistema de comunicaciones simulado. Finalmente en la implementación se llevó a cabo la creación y descarga del archivo de programación, sobre hardware reconfigurable (FPGA), del sistema de comunicaciones simulado.

\section{A. Modelado}

En la Fig. 1 se presenta el modelo de referencia para el sistema de comunicaciones banda base con modulación 16/64 QAM, los bloques relacionados en el modelo son descritos a continuación:

\section{1) Fuente de información}

Genera una secuencia de bits con una velocidad de transmisión de datos determinada. Se asume que las probabilidades de los bits generados, sea cero o uno, son equiprobables y estadísticamente independientes.
2) Divisor de datos

Distribuye los bits generados por la fuente de información a través las ramas I y Q que representan las componentes en fase (I) y cuadratura (Q) de la modulación QAM.

3) Conversor de 2 a $\mathrm{M}$ niveles

Asocia, a los bits de las ramas I y Q, los símbolos complejos correspondientes de acuerdo al mapeo establecido y genera una forma de onda rectangular tanto en la rama I como en la rama Q, por cada símbolo complejo.

4) Normalizador de potencia

Altera el nivel de amplitud de las señales proporcionadas por el conversor de 2 a $\mathrm{M}$ niveles con el fin de obtener una potencia promedio igual a uno, en cada una de las ramas (I y Q).

\section{5) Canal AWGN complejo}

Se asume un canal de comunicaciones no limitado en banda que adiciona ruido AWGN complejo a las señales provenientes del normalizador de potencia.

\section{6) Desnormalizador de potencia}

Altera el nivel de amplitud de las señales recibidas con el fin de revertir la normalización de potencia realizada en el transmisor.

\section{7) Decisor}

Establece, con base en un criterio de decisión, el valor del símbolo complejo que le corresponde a cada par de niveles de las señales de las ramas I y Q proporcionadas por el normalizador de potencia.

En el caso de una fuente que emite símbolos independientes y equiprobables, el criterio de decisión de mínima probabilidad de error corresponde al criterio de mínima distancia, el cual consiste en determinar el símbolo de referencia que se encuentra más cercano geométricamente al símbolo obtenido después del canal [6].

\section{8) Conversor de $\mathrm{M}$ a 2 niveles}

Identifica cada uno de los símbolos complejos que representan conjuntamente las señales proporcionadas por el decisor y genera por cada símbolo un conjunto de bits tanto en la rama I como en la rama Q de acuerdo al mapeo utilizado en el modulador. 


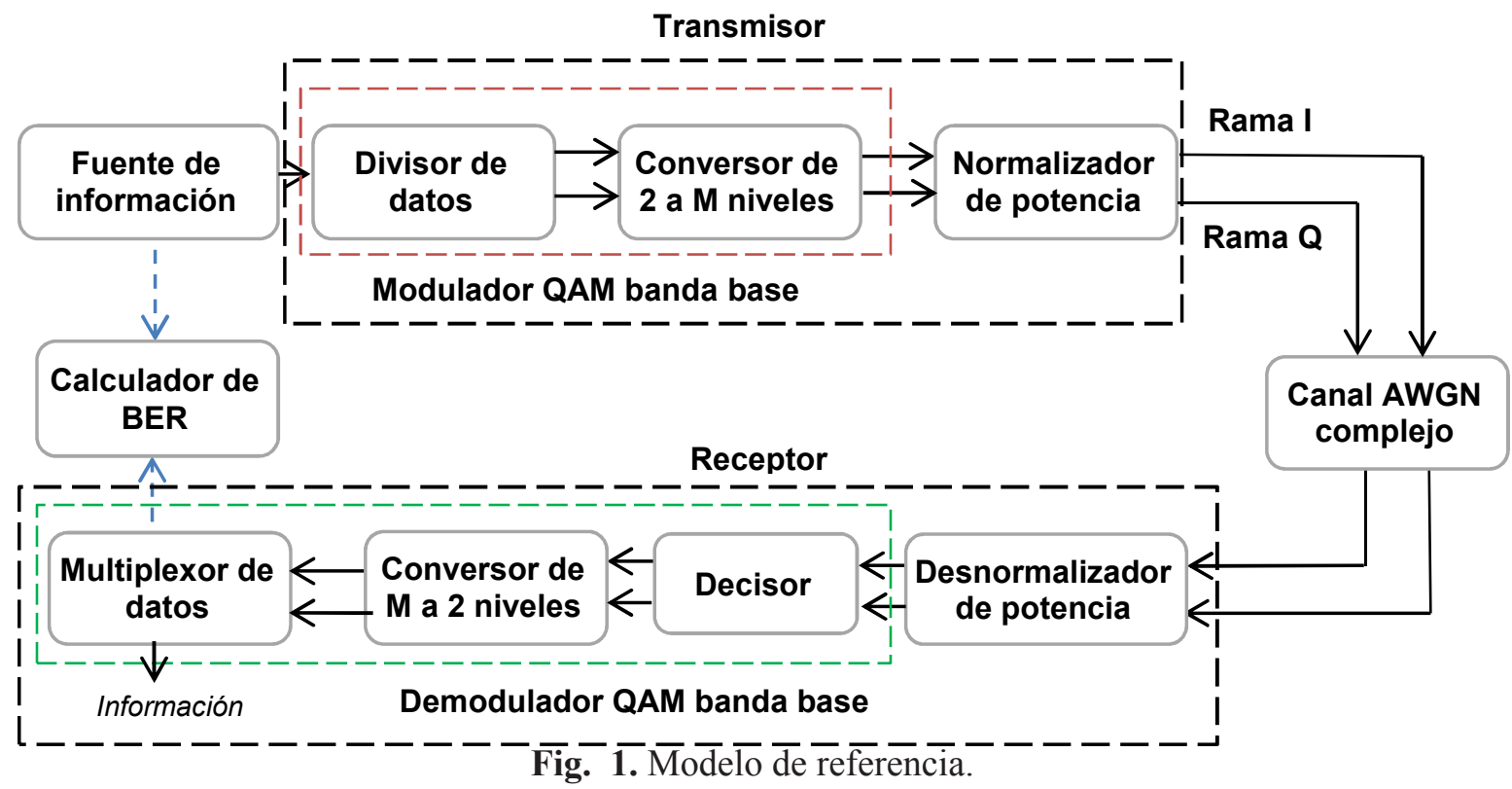

9) Multiplexor de datos

Agrupa los bits de las ramas I y Q en una sola secuencia, generando la secuencia de bits recuperada o también denominada señal demodulada.

10) Calculador de BER

Con el fin de evaluar la calidad de transmisión del sistema de comunicaciones banda base, este bloque obtuvo el número de bits errados de la señal demodulada y lo dividió entre la cantidad total de bits transmitidos para obtener el valor de BER correspondiente a un nivel de ruido determinado por el valor de la relación energía de bit a densidad espectral de potencia de ruido $(\mathrm{Eb} / \mathrm{No})$.

\section{B. Simulación}

La herramienta software seleccionada para la simulación y posterior generación del archivo de programación del sistema de comunicaciones banda base con modulación 16/64 QAM fue System Generator ${ }^{\circledR}$ del paquete de diseño del Ambiente de Software Integrado (ISE, Integrated Software Environment), cuya elección se debió a que no se requiere experiencia previa con metodologías de diseño sobre FPGAs de Xilinx ${ }^{\circledR}$, se habilita el uso del entorno de modelado de Simulink ${ }^{\circledR}$ para el diseño basado en FPGA y los diseños son procesados por el entorno de Simulink ${ }^{\circledR}$ utilizando un conjunto de bloques específico de Xi$\operatorname{linx}{ }^{\circledR}[7]$.

1) Modelo en System Generator ${ }^{\circledR}$

A partir del modelo de referencia plateado en el modelado, se construyó el modelo en System Genera- tor ${ }^{\circledR}$ del sistema de comunicaciones banda base con modulación 16/64 QAM.

La fuente de información de la Fig. 2 fue construida a partir de un Registro de Desplazamiento con Realimentación Lineal (LFSR, Linear Feedback Shift Register) que generó una secuencia de bits pseudoaleatoria.

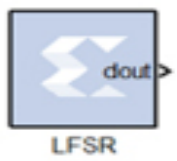

Fig. 2. Fuente de información.

El diseño del divisor de datos de la Fig. 3 implicó el arreglo de dos Mux controlados por un Counter para distribuir los bits por las ramas I y Q.

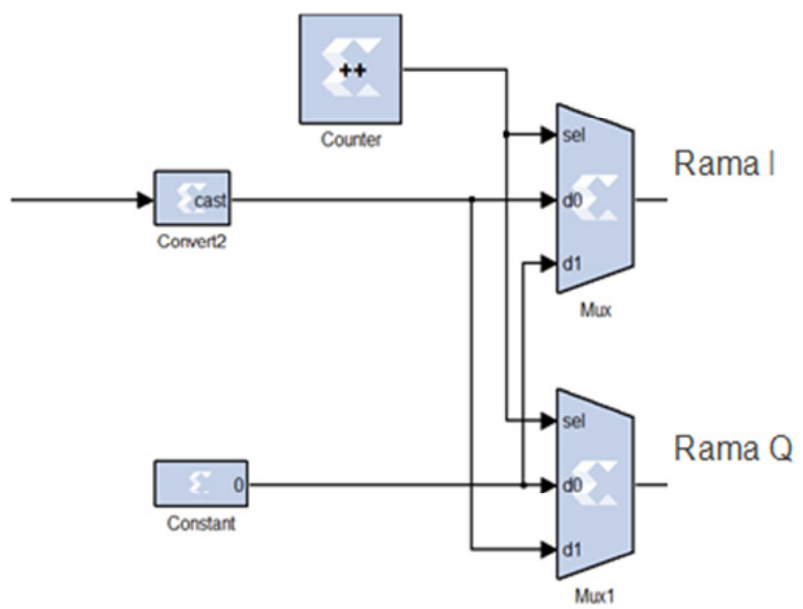

Fig. 3. Divisor de datos. 
El conversor de 2 a $\mathrm{M}$ niveles de la Fig. 4 requirió, además de un Delay en la rama I y un Serial to Parallel seguido de un Down Sample en las ramas I y Q para eliminar efectos no deseados e introducidos por del divisor de datos, un Mcode (programado en lenguaje Matlab®) que mapeó los bits a sus correspondientes símbolos complejos y generó las formas de onda rectangulares respectivas.

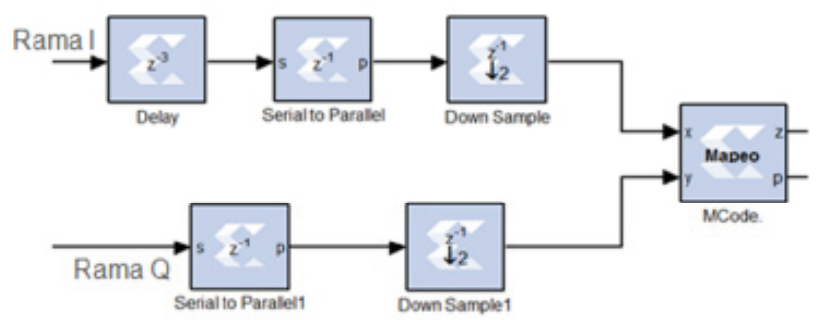

Fig. 4. Conversor de 2 a $\mathrm{M}$ niveles.

El normalizador de potencia de la Fig. 5 implicó el uso de un Mult y un Constant, tanto en la rama I como en la rama $\mathrm{Q}$, para realizar el producto entre el factor de normalización de potencia correspondiente y las señales proporcionadas por el Mcode.
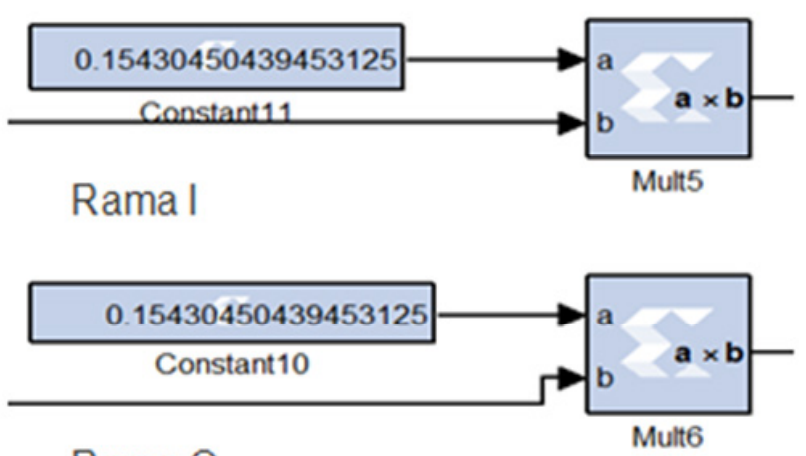

Rama $\mathrm{O}$

Fig. 5. Normalizador de potencia.

La construcción del canal AWGN complejo de la Fig. 6 se realizó a través de dos canales AWGN no correlacionados, uno en la rama I y otro en la rama $\mathrm{Q}$, que representaron las componentes en fase (I) y cuadratura (Q) del ruido AWGN complejo; para tal fin, se utilizó en cada rama (I y Q), un Generador de Ruido Blanco Gaussiano (WGNG, White Gaussian Noise Generator) que generó ruido AWGN con un nivel de potencia normalizado.

Con el propósito de controlar la cantidad de ruido suministrada por los WGNGs de las ramas I y Q, las señales de salida de estos elementos fueron multipli-

2 Efectos no deseados tales como: desincronización y presencia de ceros ajenos en las secuencias de bits de las ramas $I$ y $Q$. cadas, a través de un Mult, por un factor de ruido , configurado en un Constant, que permitió generar el valor de potencia de ruido normalizado correspondiente a un determinado valor de la relación energía de bit a densidad espectral de potencia de ruido $(\mathrm{Eb} /$ No). Finalmente un AddSub tanto en la rama I como en la rama $\mathrm{Q}$, adicionó el valor de potencia de ruido deseado, a las señales proporcionadas por el normalizador de potencia.

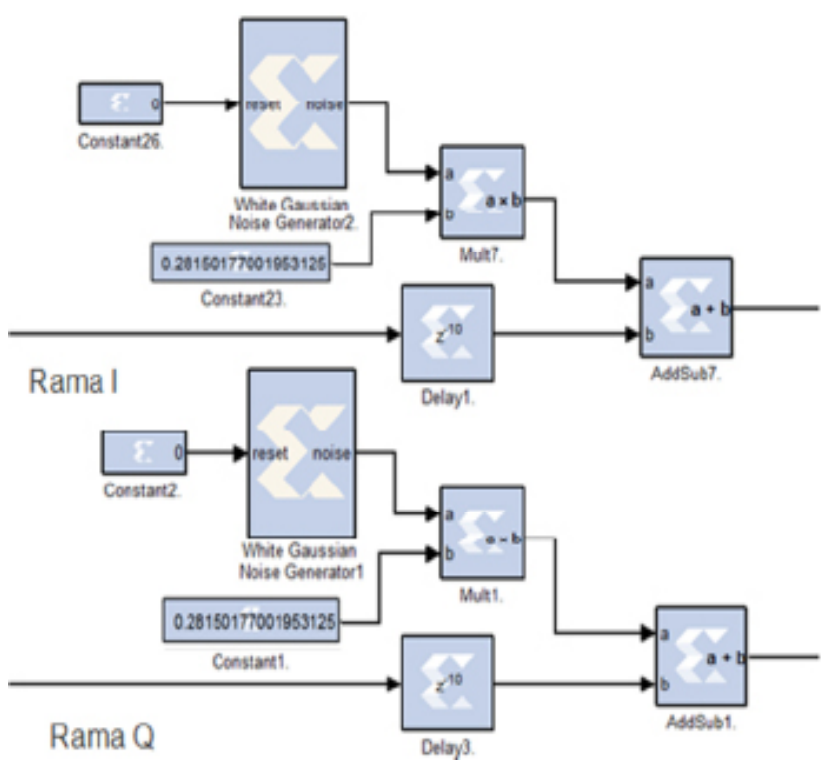

Fig. 6. Canal AWGN complejo.

El Desnormalizador de potencia de la Fig. 7 implicó el uso de un Mult y un Constant, tanto en la rama I como en la rama $\mathrm{Q}$, para realizar el producto entre el valor inverso del factor de normalización aplicado en el transmisor y las señales proporcionadas por el Mcode.
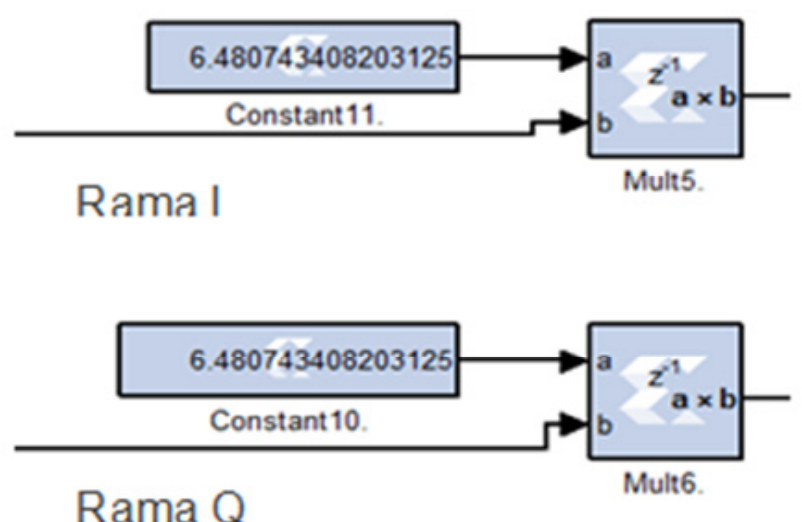

Fig. 7. Desnormalizador de potencia.

3 Para los casos de las modulaciones 16 y 64 QAM, los factores de normalización de potencia corresponden a $1 / \sqrt{ } 10$. y $1 / \sqrt{4} 42$, respectivamente.

4 Para una Eb/No dada, el factor de ruido corresponde con el valor de la desviación estándar ( $\sigma$ ), que una vez multiplicado por la señal de salida del $W G N G$ permite generar un nivel de potencia de ruido normalizado igual al valor de la varianza $(\sigma 2)$. 
El decisor de la Fig. 8 fue construido a partir de un Mcode (programado en lenguaje Matlab ${ }^{\circledR}$ ) que estableció rangos de decisión o valores umbrales para determinar, con base en el criterio de decisión de distancia mínima, a qué valores pertenecían los niveles de las señales proporcionadas por el desnormalizador de potencia.

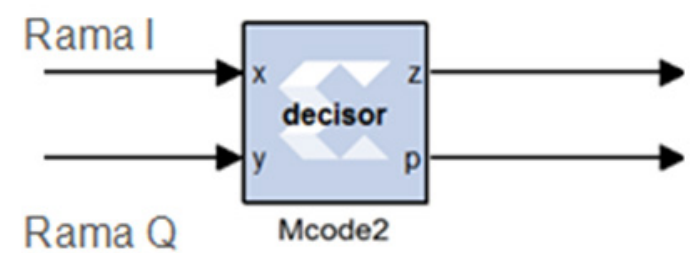

Fig. 8. Decisor.

El conversor de $\mathrm{M}$ a 2 niveles de la Fig. 9 requirió un Mcode (programado en lenguaje Matlab®), que demapeó los símbolos complejos a sus correspondientes señales de salida, además de un Delay en la rama Q y un Parallel to Serial seguido de un Up Sample en las ramas I y Q para introducir los respectivos efectos eliminados por el conversor de 2 a $\mathrm{M}$ niveles.

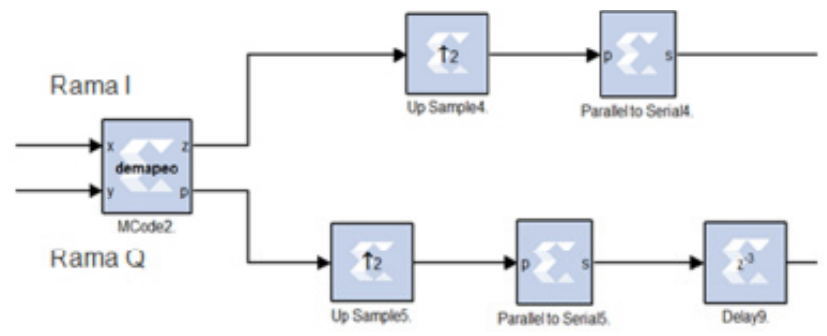

Fig. 9. Conversor de a 2 niveles.

El multiplexor de la Fig. 10 fue construido a partir de un Mux controlado por un Counter para combinar las secuencias de bits de las ramas I y Q, proporcionadas por el conversor de $\mathrm{M}$ a 2 niveles, en una sola secuencia y así obtener la secuencia de bits recuperada o también denominada señal demodulada.

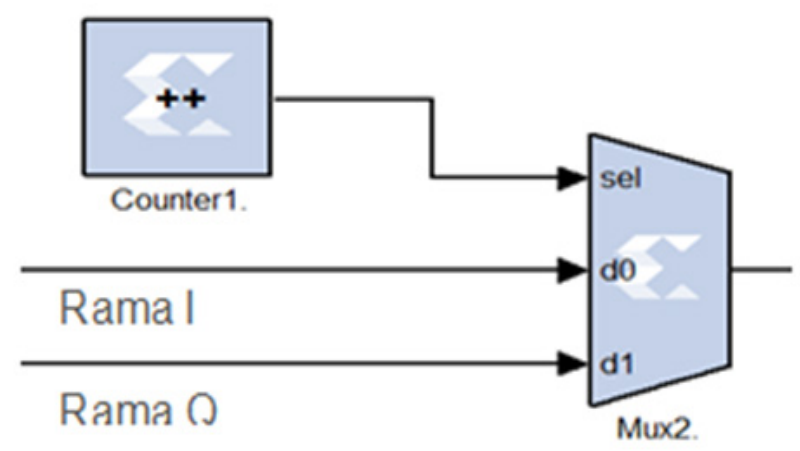

Fig. 10. Multiplexor de datos.

5 Dado que tanto el decisor como el conversor de M a 2 niveles requirieron un Mcode, se utilizó un único Mcode con la lógica de ambos bloques.
La construcción del calculador de BER de la Fig. 11 requirió de un Delay para la sincronización de la señal transmitida con la señal demodulada; un Counter para controlar el número total de bits transmitidos; un Accumulator para almacenar el número de bits errados de la señal demodulada; un Mcode (programado en lenguaje Matlab $\left.{ }^{\circledR}\right)$ para calcular el valor de la BER y un Dual Port Ram para almacenar este valor.

Los anteriores elementos corresponden a los más importantes del diseño de la BER, sin embargo también se utilizaron Converts que cambiaron el tipo de dato entre dos elementos, Constants que habilitaron e indicaron los puertos de lectura y escritura del Dual Port Ram y Relationals que habilitaron o deshabilitaron los elementos utilizados.

\section{2) Validación}

La validación del correcto funcionamiento del modelo en System Generator ${ }^{\circledR}$ se realizó por medio de la comparación de las curvas de desempeño obtenidas a partir del sistema de comunicaciones banda base con modulación 16/64 QAM simulado en System Generator ${ }^{\circledR}$ y las curvas de desempeño obtenidas a partir de un modelo en Simulink ${ }^{\circledR}$. Para tal efecto se consideró la transmisión de 32768 bits, el uso del mapeo Gray, un canal AWGN no limitado en banda y el criterio de decisión de mínima distancia.

Las Fig. 12 y Fig. 13 muestran las curvas de desempeño del sistema de comunicaciones banda base con modulación 16/64 QAM simulado en Simulink ${ }^{\circledR}$ y System Generator ${ }^{\circledR}$. Las superposiciones de las curvas de desempeño permitieron asegurar que el modelo propuesto fue consistente y por ende apto para el estudio del desempeño a nivel de implementación sobre hardware reconfigurable (FPGA). 


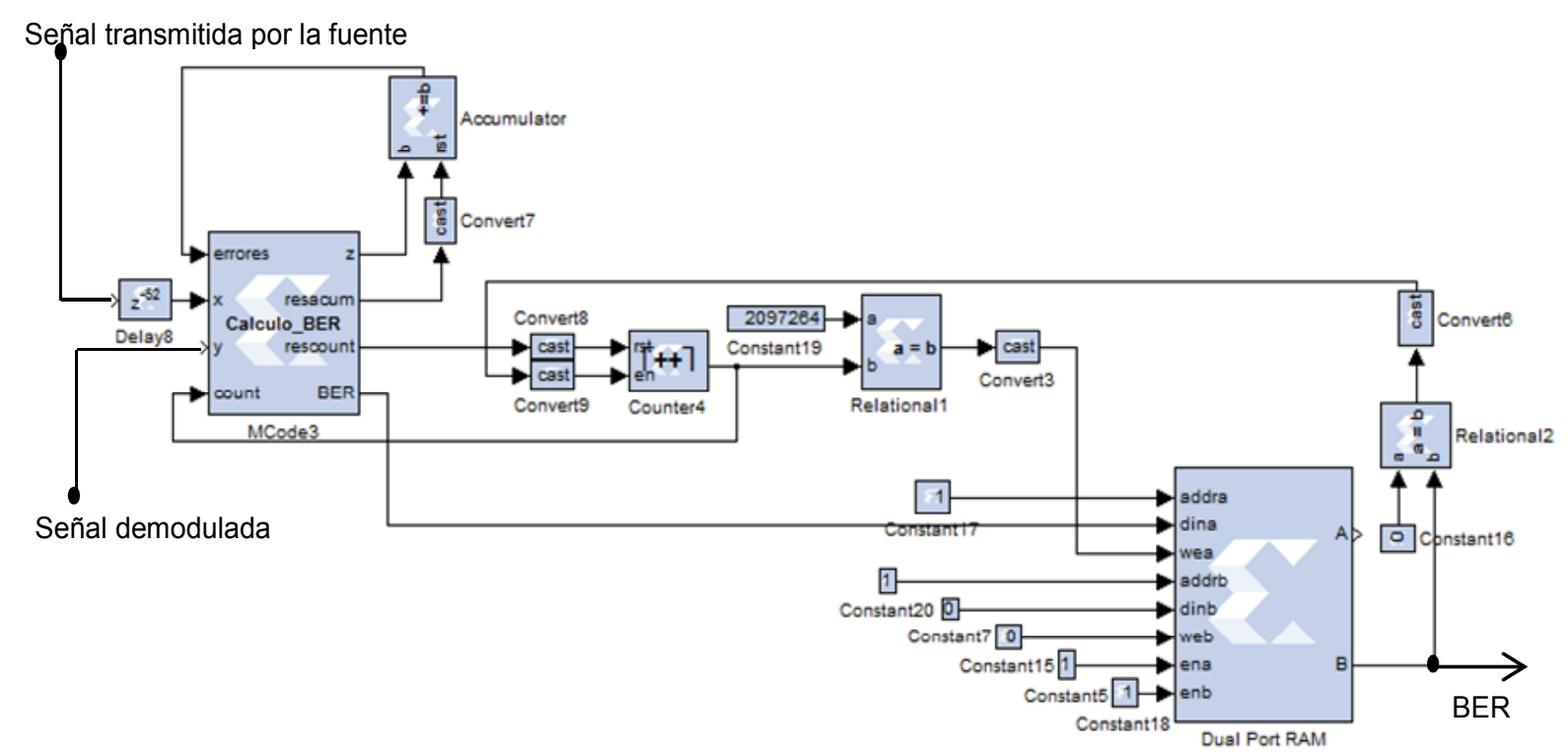

Fig. 11. Calculador de BER.

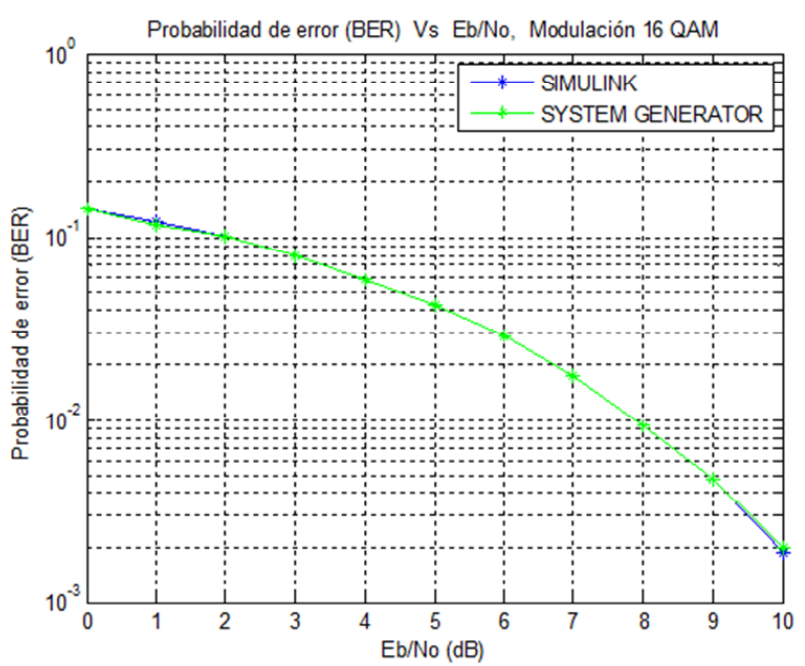

Fig. 12. Curvas de desempeño a partir de Simulink ${ }^{\circledR}$ y System Generator ${ }^{\circledR}$, modulación 16 QAM.

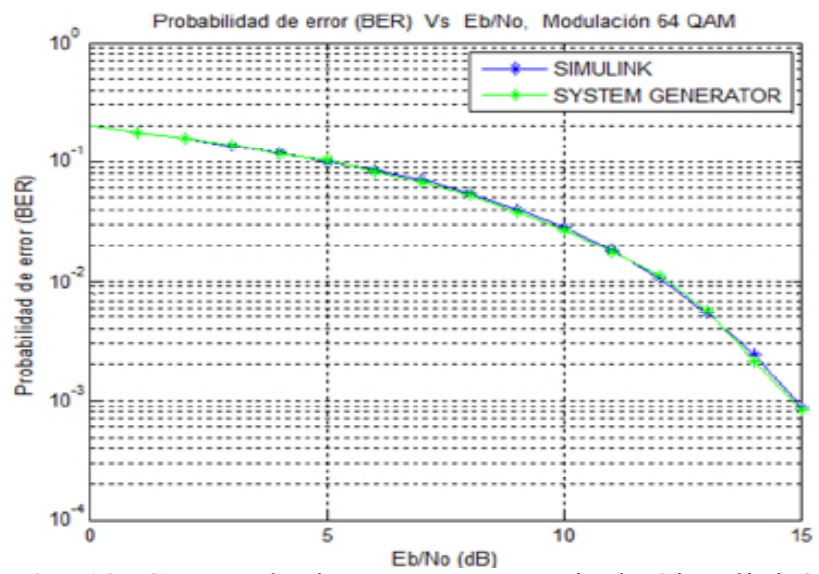

Fig. 13. Curvas de desempeño a partir de Simulink ${ }^{\circledR}$ y System Generator ${ }^{\circledR}$, modulación 64 QAM.
C. Implementación

La implementación del sistema de comunicaciones banda base con modulación 16/64 QAM se llevó a cabo sobre un FPGA Spartan 6 de Xilinx ${ }^{\circledR}$; para lo cual, la generación del archivo de programación del FPGA se obtuvo a través de System Generator ${ }^{\circledR}$. Finalmente, la descarga del archivo sobre el FPGA se realizó por medio de la herramienta de programación iMPACT del paquete de diseño ISE.

\section{RESULTADOS Y DISCUSIÓN}

Con el objetivo principal de analizar el desempeño del sistema de comunicaciones banda base con modulación 16/64 QAM implementado sobre hardware reconfigurable (FPGA), se recurrió a la variación del tipo de mapeo, además de la variación de la velocidad de transmisión de datos y la cantidad de bits transmitidos que, sin afectar el desempeño del sistema de comunicaciones, permitieran corroborar el correcto funcionamiento del hardware reconfigurable (FPGA).

\section{A.Variación Velocidad de Transmisión de Datos}

Para una cantidad de 32768 bits transmitidos y mapeo Gray, las Fig. 14 y Fig. 15 muestran los resultados de simulación en System Generator ${ }^{\circledR}$ e implementación sobre hardware reconfigurable (FPGA), respectivamente, del desempeño del sistema de comunicaciones banda base con modulación 16 QAM, con dos velocidades de transmisión de datos: 500 Kbps y 10 Mbps. Aunque las gráficas mostradas corresponden única- 
mente a la modulación 16 QAM, los resultados tuvieron el mismo comportamiento para la modulación 64 QAM.

Dada la superposición de las curvas de desempeño de las Fig. 14 y Fig. 15, se pudo verificar la independencia del sistema de comunicaciones banda base con modulación 16/64 QAM ante las variaciones de este parámetro, ello en virtud a que el sistema de comunicaciones no consideró efecto de multitrayectoria o corrimiento en frecuencia y no consideró limitación en ancho de banda que hiciera que el canal se comportara de manera selectiva en frecuencia; con lo cual pudo obviarse la adición de una etapa de ecualización y la velocidad de transmisión de datos no dio lugar a Interferencia Intersimbolo (ISI, Intersymbol Interference), un factor determinante en la demodulación.

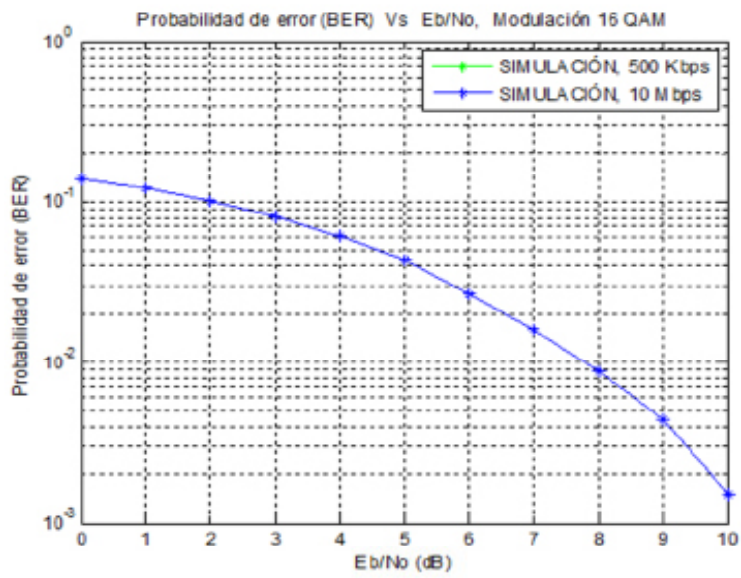

Fig. 14. Curvas de desempeño para velocidades de transmisión de datos $500 \mathrm{Kbps}$ y $10 \mathrm{Mbps}$, simulación en System Generator ${ }^{\circledR}$ con modulación 16 QAM.

\section{B. Variación Tipo de Mapeo}

Para una cantidad de 32768 bits transmitidos y una velocidad de transmisión de datos de $500 \mathrm{Kbps}$, las Fig. 16 y Fig. 17 muestran los resultados de simulación en System Generator ${ }^{\circledR}$ del desempeño del sistema de comunicaciones banda base con modulación 16/64 QAM, para dos tipos de mapeo: Gray y Binario; asimismo las Fig. 18 y Fig. 19 muestran los resultados de implementación sobre hardware reconfigurable (FPGA).

De los dos tipos de mapeo utilizados, la Fig. 18 permitió observar que en el caso de la modulación 16 QAM del sistema de comunicaciones implementado sobre hardware reconfigurable (FPGA), para una

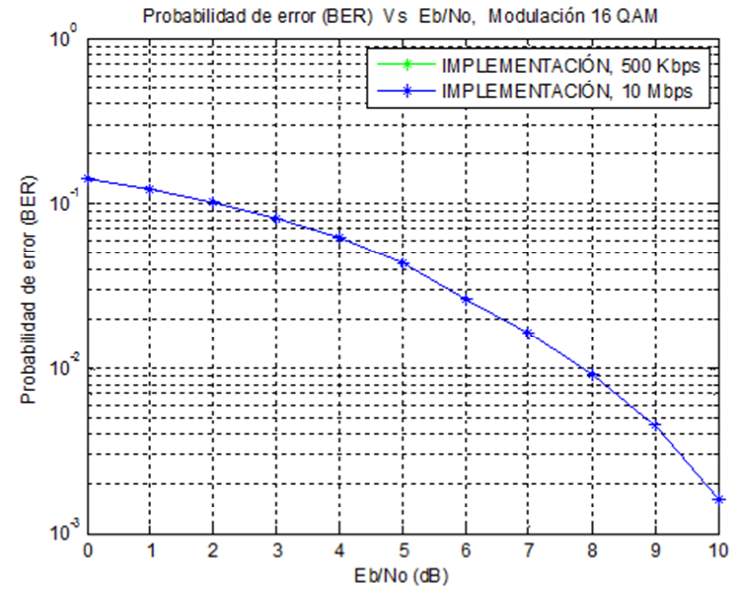

Fig. 15. Curvas de desempeño para velocidades de transmisión de datos $500 \mathrm{Kbps}$ y $10 \mathrm{Mbps}$, implementación con modulación 16 QAM.

BER objetivo de 10-2, la ganancia de codificación del mapeo Gray frente al Binario fue de $0.41 \mathrm{~dB}$, lo correspondiente a un ahorro de energía aproximado al 5.22\%; de la misma manera, la Fig. 19 en el caso de la modulación 64 QAM del sistema de comunicaciones banda base implementado sobre hardware reconfigurable (FPGA), mostró que para una BER objetivo de 10-2, la ganancia de codificación del mapeo Gray frente al Binario fue de $0.63 \mathrm{~dB}$, lo correspondiente a un ahorro de energía aproximado al 5.25\%.

Lo anterior en razón a que la diferencia entre símbolos vecinos correspondió a un bit para el mapeo Gray, caso contrario al mapeo Binario, donde se encontraron variaciones de más de un bit (entre símbolos vecinos) lo cual dio lugar a la posibilidad de mayor cantidad de bits errados por símbolo errado que se demoduló.

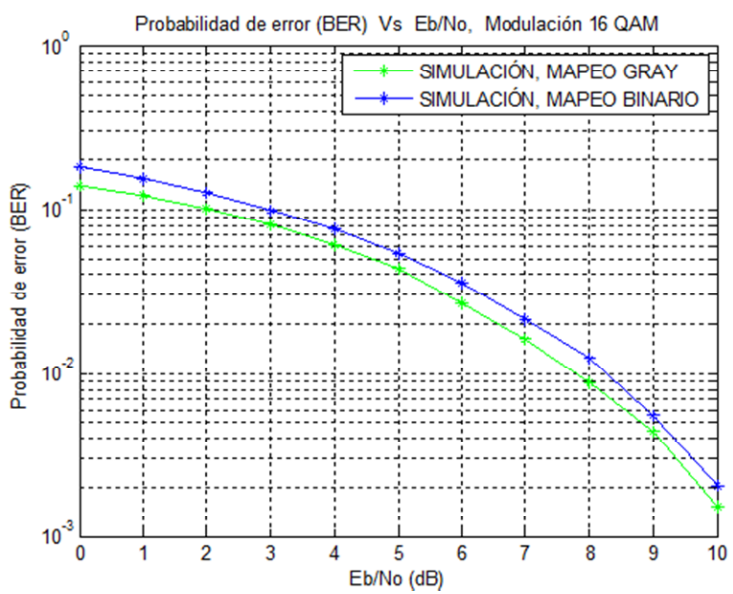

Fig. 16. Curvas de desempeño mapeos Gray y Binario, simulación en System Generator ${ }^{\circledR}$ con modulación 16 QAM. 


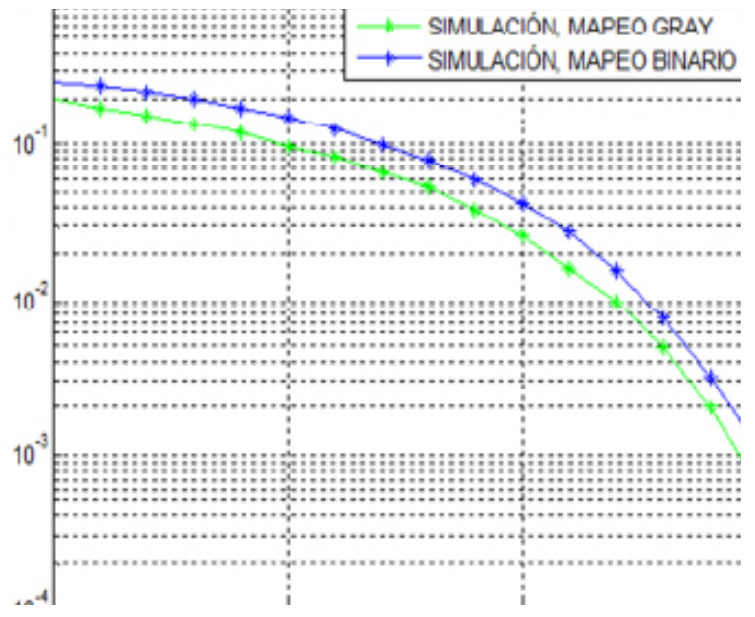

Fig. 17. Curvas de desempeño mapeos Gray y Binario, simulación en System Generator ${ }^{\circledR}$ con modulación 64 QAM.

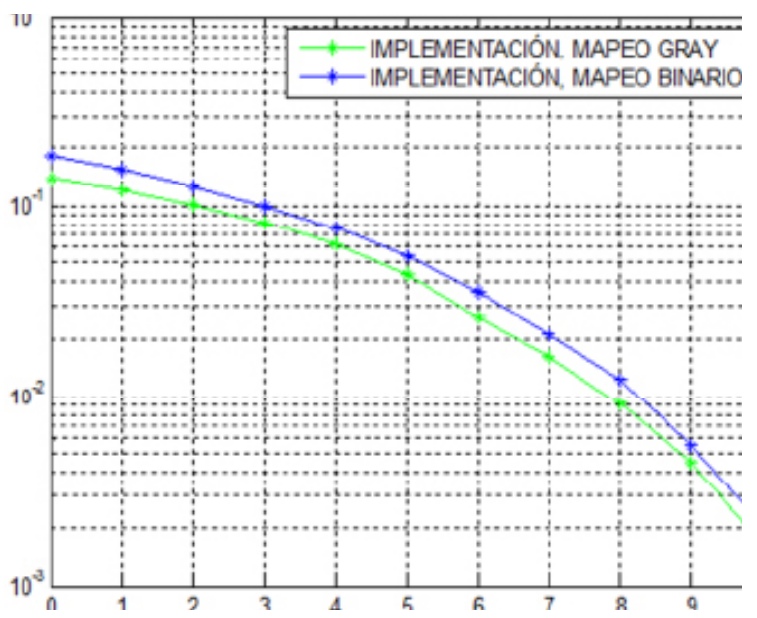

Fig. 18. Curvas de desempeño mapeos Gray y Binario, implementación con modulación 16 QAM.

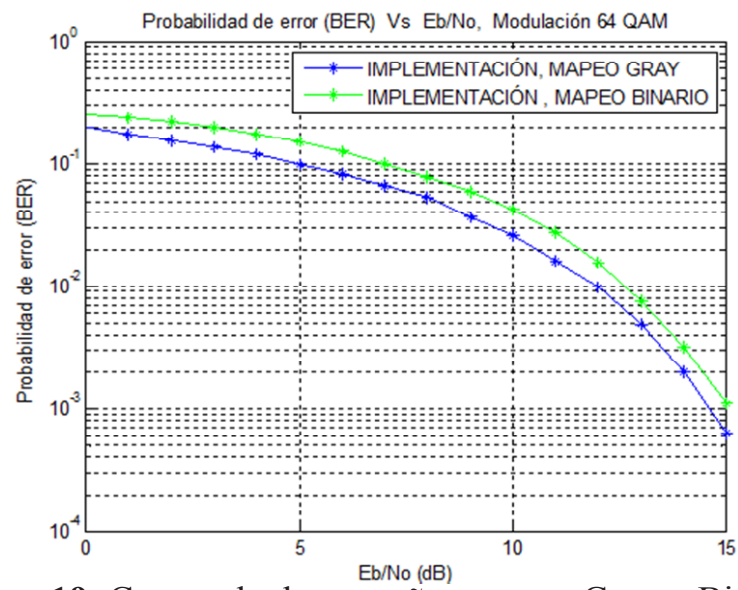

Fig. 19. Curvas de desempeño mapeos Gray y Binario, implementación con modulación 64 QAM.

\section{Variación Cantidad de Bits Transmitidos}

Para una velocidad de transmisión de datos de 500 Kbps y mapeo Gray, las Fig. 20 y Fig. 21 muestran los resultados de simulación en System Generator ${ }^{\circledR}$ e implementación sobre hardware reconfigurable (FPGA), respectivamente, del desempeño del sistema de comunicaciones banda base con modulación 16 QAM, para dos cantidades de bits transmitidos: 32768 y 1048576.

Los resultados tuvieron la misma tendencia para la modulación 64 QAM.

Se pudo apreciar cómo consistentemente las gráficas presentaron variaciones en los últimos valores de BER para un valor de $\mathrm{Eb} / \mathrm{No}$ dado, respecto a la curva teórica de Bertool $\AA$, tal como lo presentan las Fig. 22 y Fig. 23; de este modo se estableció que en la cercanía al valor de BER mínimo alcanzable diferente de cero, cualquier par de valores de Eb/No contó con una diferencia mínima en materia de cantidad de errores, por lo cual, pequeñas cantidades de bits errados alteraron considerablemente el valor de la BER correspondiente a cada Eb/No y ocasionaron las desviaciones apreciadas en las curvas de desempeño ilustradas.

Adicionalmente, las Fig. 22 y Fig. 23 muestran cómo los últimos valores de BER de la transmisión de 32768 bits fueron corregidos por la transmisión de 1048576 bits, debido a que para esta última transmisión, en la cercanía al valor de BER mínimo alcanzable diferente de cero correspondiente a la transmisión de 32768 bits, pequeñas cantidades de bits errados no alteraron el valor de BER; por lo tanto, se omitieron los últimos valores de BER que presentaron variación en todas las cuervas de desempeño, ya que no representaron un comportamiento como tal del sistema de comunicaciones banda base propuesto, sino que dependieron de un parámetro específico (cantidad de bits transmitidos) y dichas variaciones se corrigieron incrementando el valor de este parámetro.

De acuerdo a lo plateado, una mayor cantidad de bits transmitidos implicó una mayor precisión en la BER calculada, y aunque los sistemas de comunicaciones banda base propuestos soportaron la transmisión de una mayor cantidad de bits, la carga computacional fue alta y los resultados obtenidos con 1048576 bits 
fueron suficientes para el cumplimiento de los objetivos planteados.

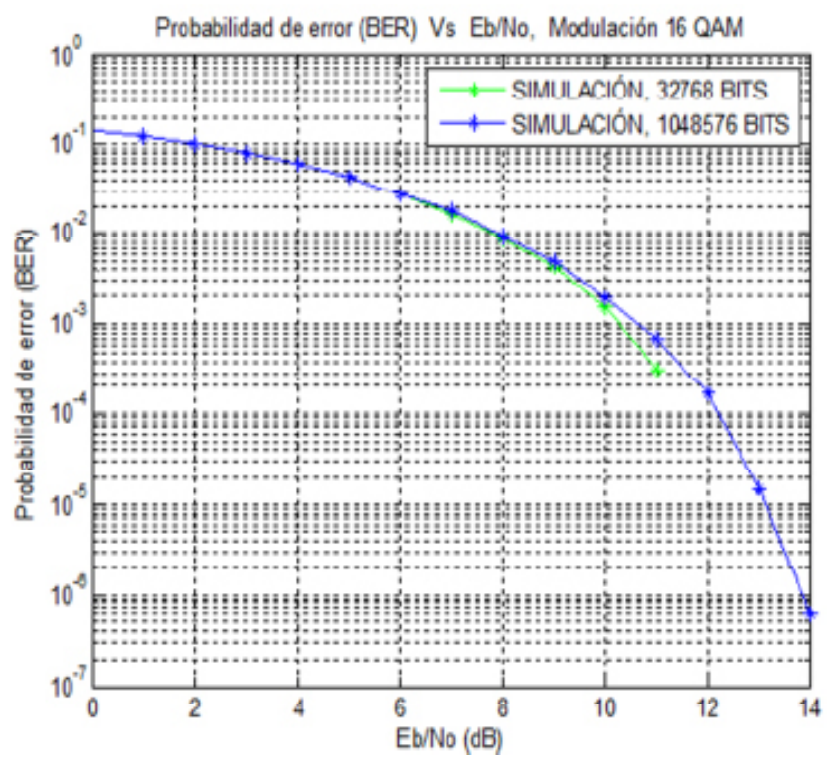

Fig. 20. Curvas de desempeño para transmisión de 32768 y 1048576 bits, simulación en System Generator ${ }^{\circledR}$ con modulación 16 QAM.

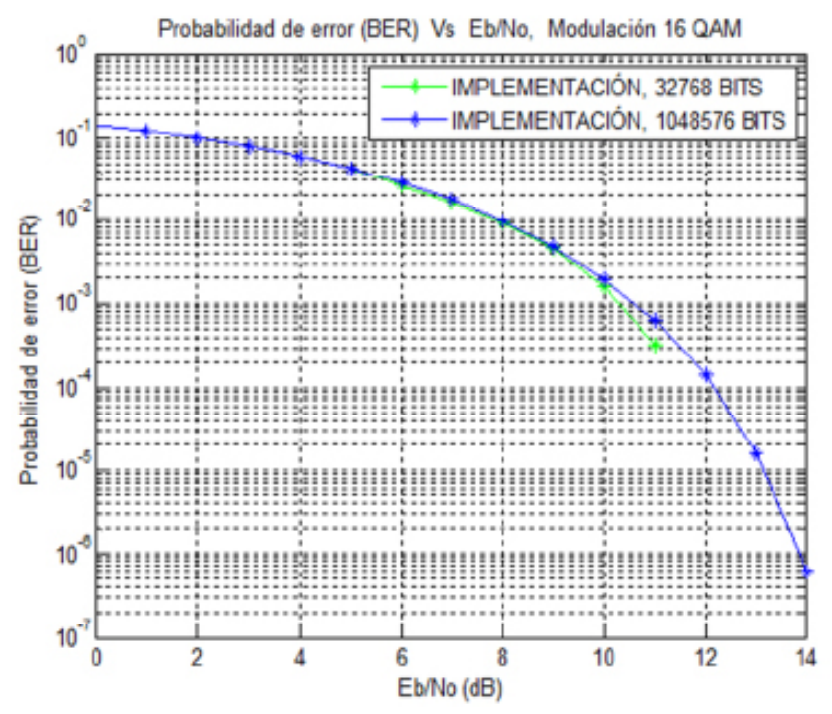

Fig. 21. Curvas de desempeño para transmisión de 32768 y 1048576 bits, implementación con modulación 16 QAM

6 Tanto 32768 como 1048576 corresponden a valores que son potencias de 2, dado que el calculador de BER cuenta con la restricción de procesar cantidades de bits con esta característica.

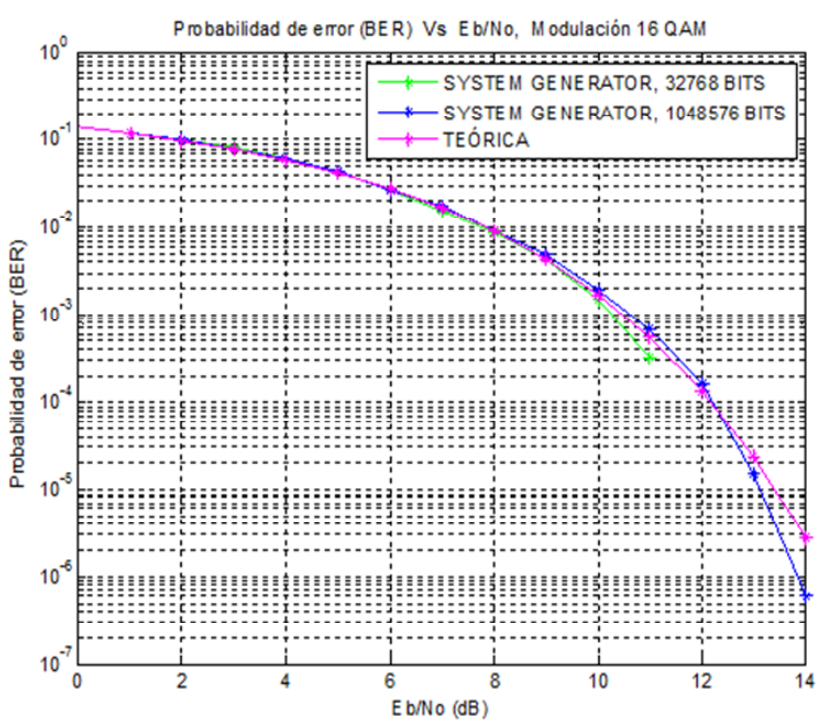

Fig. 22. Curvas de desempeño para transmisión de 32768 y 1048576 bits, simulación en System Generator® con modulación 16 QAM.

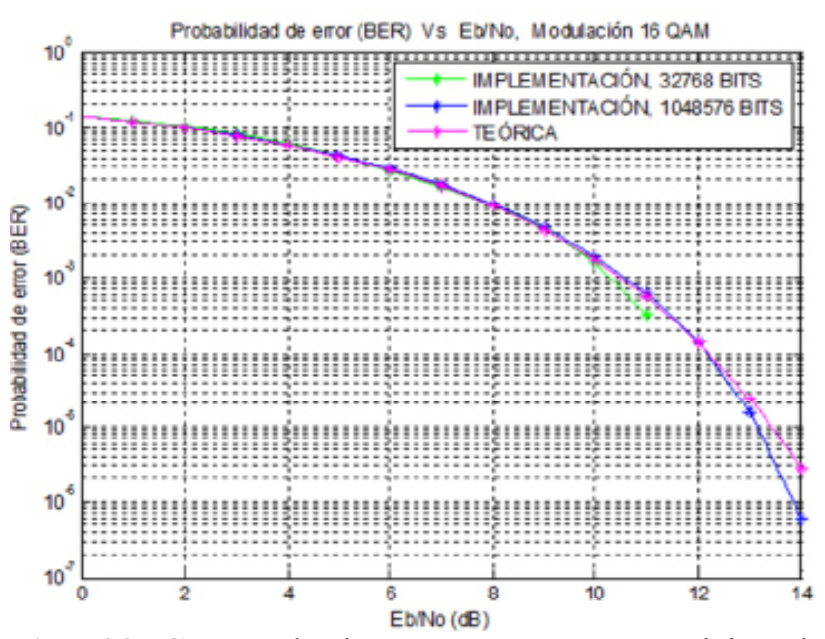

Fig. 23. Curvas de desempeño para transmisión de 32768 y 1048576 bits, implementación con modulación 16 QAM.

\section{Estudio de Curvas de Desempeño}

Teniendo en cuenta lo planteado en las discusiones anteriores, a efectos de obtener curvas de desempeño con mejores condiciones y por ende lo más similares posible a la curva de desempeño teórica, para las siguiente discusiones se consideró el uso del mapeo Gray y la transmisión de 1048576 bits así como una velocidad de transmisión de datos igual a $10 \mathrm{Mbps}$; que bien pudo haber sido $500 \mathrm{Mbps}$, dado que su valor no afecta el desempeño del sistema de comunicaciones banda base. 
1) Comparación resultados de simulación e implementación

Las Fig. 24 y Fig. 25 muestran la comparación de las curvas desempeño del sistema de comunicaciones banda base con modulación 16/64 QAM obtenidas a partir de simulación en System Generator ${ }^{\circledR}$ y a partir de implementación sobre hardware reconfigurable (FPGA), donde se apreció una clara correspondencia y estrecha relación entre los resultados obtenidos, en cada una de las figuras, dada la superposición presentada entre las curvas de desempeño; de este modo, se verificó que la implementación generó resultados coherentes de acuerdo a los modelos planteados.

2) Comparación resultados de implementación y teóricos

Las Fig. 26 y Fig. 27 muestran los resultados obtenidos a partir de implementación comparados con las curvas de desempeño teóricas ofrecidas por Bertool ${ }^{\circledR}$. Para este par de imágenes se pudo apreciar también una clara correspondencia y estrecha relación entre cada par de curvas de desempeño (implementación y teórica), por lo cual se confirmó que tanto a nivel de simulación e implementación, el sistema de comunicaciones banda base con modulación 16/64 QAM presentó un comportamiento óptimo al replicar de manera muy similar las curvas de desempeño teóricas ofrecidas por Bertool ${ }^{\circledR}$.

3) Comparación resultados de implementación modulación 16 QAM y 64 QAM

La Fig. 28 muestra simultáneamente las curvas de desempeño del sistema de comunicaciones banda base con modulación 16/64 QAM obtenidas a partir de implementación sobre hardware reconfigurable (FPGA), donde se corroboró que para menor cantidad de estados (16 QAM) se presentó mayor robustez frente al ruido, lo cual se tradujo en menor requerimiento de Eb/No para una misma BER. Consecuentemente, se pudo apreciar que pese a la utilización del mismo tipo de mapeo, la curva de desempeño correspondiente a la modulación 16QAM obtuvo un ahorro energía aproximado de $49.81 \%$ para una BER objetivo de $10^{-2}$.

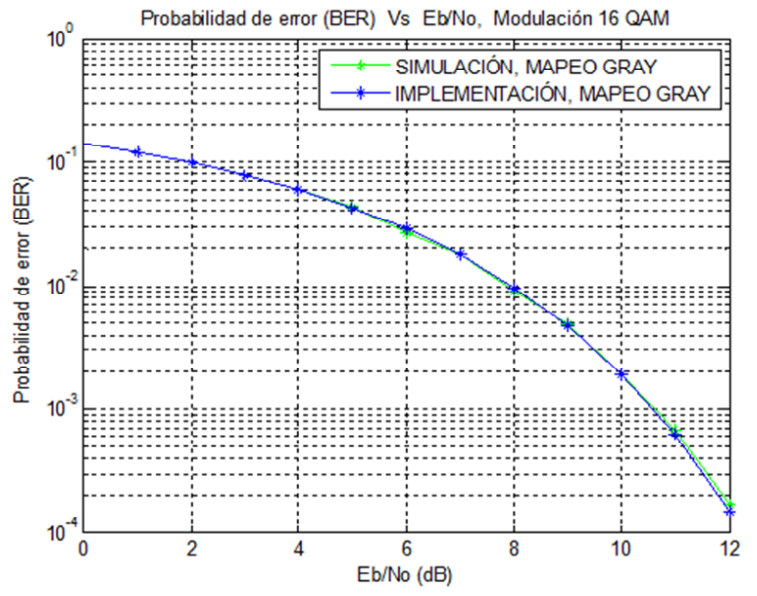

Fig. 24. Comparación curvas de desempeño, simulación en System Generator ${ }^{\circledR}$ e implementación con modulación 16 QAM y mapeo Gray.

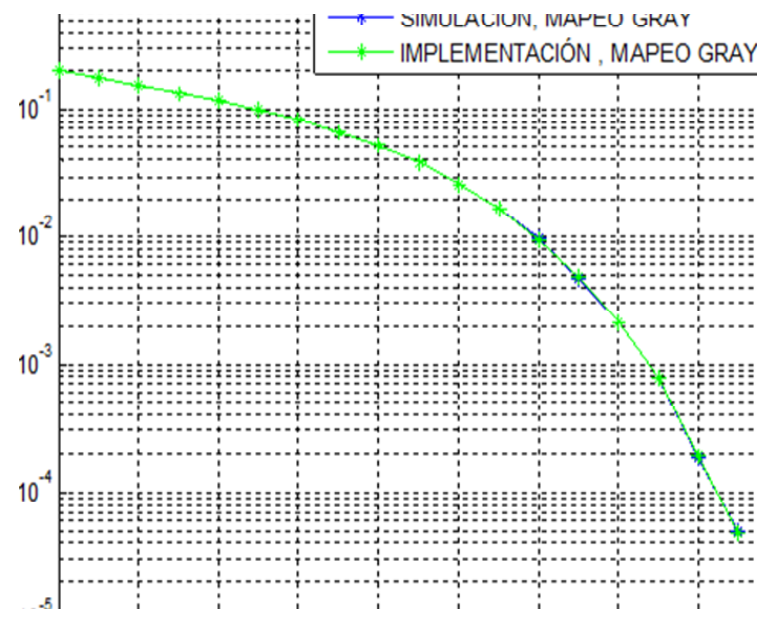

Fig. 25. Comparación curvas de desempeño, simulación en System Generator ${ }^{\circledR}$ e implementación con modulación 64 QAM y mapeo Gray.

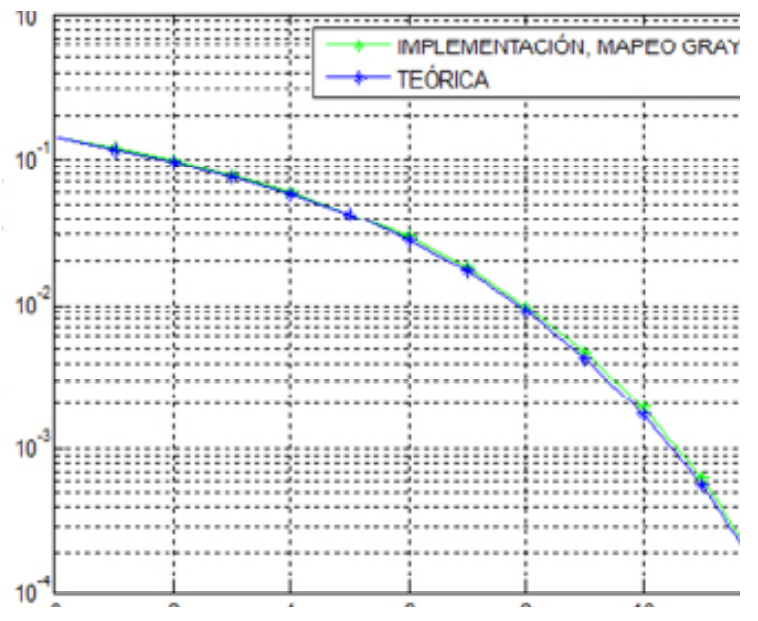

Fig. 26. Comparación curvas de desempeño, implementación y teórica con modulación 16 QAM y mapeo Gray. 


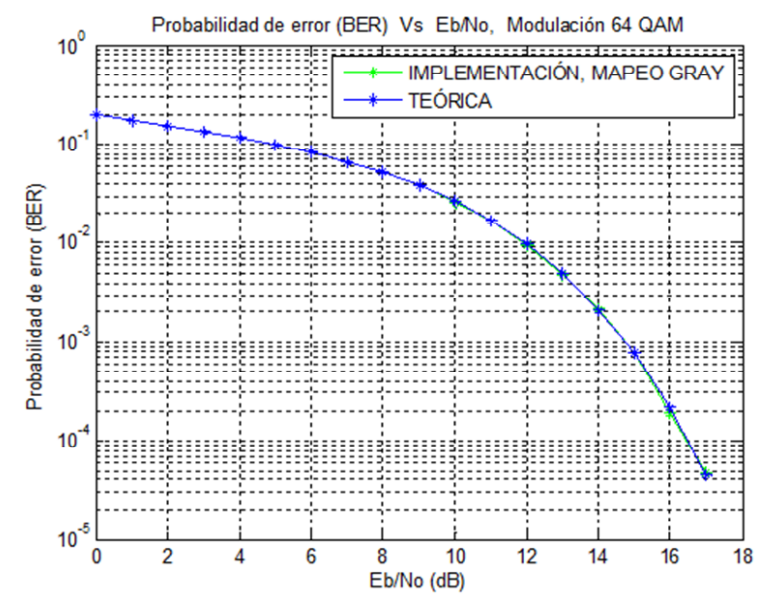

Fig. 27. Comparación curvas de desempeño, implementación y teórica con modulación 64 QAM y mapeo Gray.

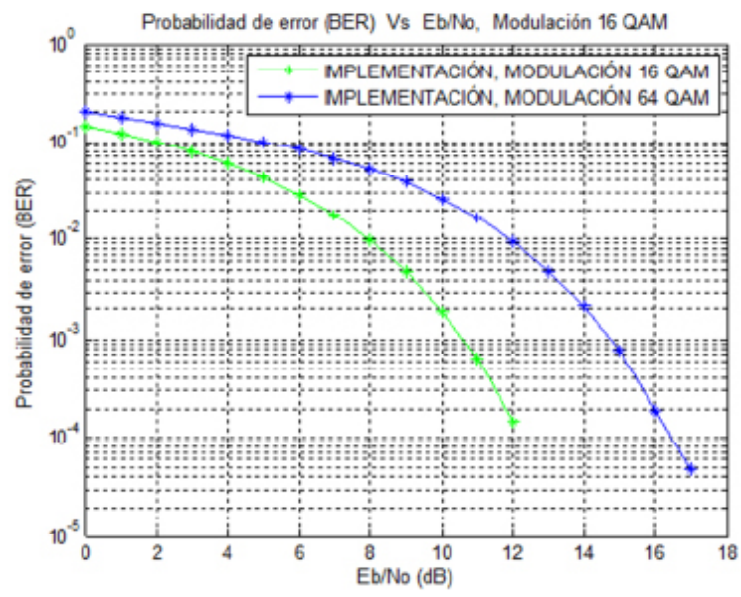

Fig. 28. Comparación cuervas de desempeño, implementación con modulación 16/64 QAM y mapeo Gray

\section{CONCLUSIONES}

1) Las simulaciones e implementaciones sobre hardware reconfigurable (FPGA) presentadas conforman la base para herramientas de apoyo a procesos de enseñanza/aprendizaje en el área de los sistemas de comunicaciones con esquemas de modulación digital.

2) A nivel de diseño, tanto 16 QAM como 64 QAM representan la misma complejidad, pues los bloques ofrecidos por System Generator ${ }^{\circledR}$ permiten modificar las configuraciones para cada una de las modulaciones sin la necesidad de alterar el modelo base, funcional para los esquemas de modulación 16 QAM y 64 QAM.
3) La cantidad de bits transmitidos incide considerablemente sobre el trazado de la curva de desempeño de un sistema de comunicaciones, donde a mayor cantidad de bits transmitidos, la tendencia estadística es mucho más clara.

4) Para una BER objetivo de 10-2 en las modulaciones 16 QAM y 64 QAM, el mapeo Gray introdujo una ganancia de codificación respecto al mapeo Binario de $0.41 \mathrm{~dB}$ y $0.63 \mathrm{~dB}$, respectivamente; lo correspondiente a un ahorro de energía de $5.22 \%$ en la modulación 16 QAM y 5.25\% en la modulación 64 QAM.

5) Para una BER objetivo de 10-2, la modulación 16 QAM con mapeo Gray obtuvo un ahorro de energía aproximado al $49.81 \%$ respecto a la modulación 64 QAM con mapeo Gray.

6) Dada la obtención de curvas de desempeño muy similares a las curvas de desempeño de simulación en System Generator ${ }^{\circledR}$, Simulink ${ }^{\circledR}$ y Bertool ${ }^{\circledR}$, el sistema de comunicaciones banda base con modulación 16/64 QAM alcanzó resultados óptimos de implementación sobre hardware reconfigurable (FPGA).

\section{AGRADECIMIENTOS}

Los autores desean agradecer los grupos de investigación I+D Grupo de Radio e InAlámbricas (GRIAL) y Grupo de Nuevas Tecnologías en Telecomunicaciones (GNTT), a la Facultad de Ingeniería Electrónica y Telecomunicaciones y a la Vicerrectoría de Investigaciones de la Universidad del Cauca por apoyar la realización del presente artículo, el cual fue realizado con base en el trabajo de grado denominado: "Análisis del Desempeño de un Sistema de Comunicaciones Banda Base con Modulación 16/64 QAM Basado en Hardware Reconfigurable", el cual forma parte del proyecto marco denominando: "Diseño e Implementación de un Prototipo de Comunicación de Datos Basado en Hardware Reconfigurable Fase 1". 


\section{BIBLIOGRAFÍA}

1. Voros, S, y Masselos, K.(2005). System Level Design of Reconfigurable Systems-on-Chip.Springer.

2. Miramá, V. (2010) "Teoría De Telecomunicaciones I, Sistemas Analógicos”. Popayán: Departamento de Telecomunicaciones, Universidad del Cauca.

3. R. Gallager,(2006) "Channels Modulations and Demodulations". En: Course Materials for 6.450 Principles of Digital Communications I. MIT OpenCourseWare. Massachusetts Institute of Technology. Descargado 24 de Enero de 2014, de http://ocw.mit.edu/

4. López, X. y Mora, C.(2006). "Análisis de Técnicas de Modulación Adaptiva en Redes Inalámbricas de Banda Ancha (ieee 802.16, wimax)”. Ciudad de México D. F.: Universidad Nacional Autónoma de México.

5. Pressman, R.(2002). Ingeniería del Software, un Enfoque Práctico. Mc Graw Hill,

6. Ramirez, J. y Moreno, J. (2009). “Análisis del Desempeño de la Modulación Wavelet", Popayán: Universidad del Cauca.

7. Xilinx ${ }^{\circledR}$, Inc.(2012). "System Generator ${ }^{\circledR}$ for DSP Getting Started Guide". Descargado 24 de Enero de 2014, de http://www.xilinx.com/support/documentation/sw_manuals/xilinx14_5/sysgen_gs.pdf 